\title{
Actitudes emprendedoras de estudiantes universitarios de primer año en Chile
}

\author{
Andrés A. Valenzuela-Keller*, Francisco A. Gálvez-Gamboa, Erik Silva-Aravena y Matias Moreno-Villagra \\ Facultad de Ciencias Sociales y Económicas, Universidad Católica del Maule, Av. San Miguel 3605, Talca, Chile. \\ (Correo-e: andresvalenzuelak@gmail.com, franziscogalvez@gmail.com, erikmanuelsilva@gmail.com, \\ matiasmorenovillagra@gmail.com)
}

* Autor a quien debe ser dirigida la correspondencia.

Recibido Ene. 22, 2021; Aceptado Mar. 18, 2021; Versión final Abr. 7, 2021, Publicado Ago. 2021

\begin{abstract}
Resumen
El objetivo de este estudio es analizar los factores que conforman la actitud emprendedora de los universitarios de primer año. Se aplica una encuesta basada en el modelo de orientación a las actitudes emprendedoras en una muestra de 322 estudiantes de las áreas de ingeniería, administración y economía. Se utiliza un análisis factorial exploratorio. Los resultados muestran que las dimensiones que interfieren en la actitud emprendedora son: 1) autoestima, 2) control personal o interno, 3) asunción de riesgo e innovación y 4) necesidad de logro, coincidente con la teoría de orientación a las actitudes emprendedoras. Se evidencia que existen diferencias atribuibles a las disciplinas, la percepción como futuro emprendedor y la capacidad percibida para lograr emprender. Se sugieren metodologías que incorporen el conocimiento del medio emprendedor a través de prácticas tempranas, vivencias o casos prácticos. Se concluye que es necesario generar diagnósticos de los estudiantes de primer año para identificar estrategias que les permitan desarrollar habilidades y competencias de emprendimiento.
\end{abstract}

Palabras clave: emprendimiento; actitud emprendedora; intención; competencia; educación

\section{Entrepreneurial attitudes of first year university students in Chile}

\begin{abstract}
The primary objective of this study is to analyze the factors that shape entrepreneurial attitude in first year university students. A survey based on the model of orientation to entrepreneurial attitudes is applied to a sample of 322 engineering, management, and economics students. An exploratory factor analysis is used for data processing. The results show that the dimensions that interfere with entrepreneurial attitude are: 1) selfesteem, 2) personal or internal control, 3) risk-taking and innovation, and 4) need for achievement. These are in agreement with the theory of entrepreneurial attitude orientation. There are also differences attributable to the field of study, the perception as a future entrepreneur, and the capacity to become an entrepreneur. Methodologies are suggested that incorporate knowledge of entrepreneurial environment through early practices, experiences, and case studies. It is concluded that it is necessary to assess first year students to identify strategies that would allow them to develop entrepreneurship skills and competences.
\end{abstract}




\section{INTRODUCCIÓN}

La misión actual de las universidades evoluciona periódicamente ajustándose a los requerimientos del medio en el cual se desenvuelve (Pinheiro et al., 2015). En este contexto, el rol de las universidades vinculada a la tercera misión es una parte trascendental para fomentar el desarrollo regional y local (Secundo et al., 2017). Este movimiento propone que las universidades sean un actor del desarrollo local, y que no sólo se enfoquen en sus dos principales misiones que son la enseñanza y la investigación (Zomer y Benneworth, 2011). Por diferentes razones, las universidades se han aproximado a esta tercera misión, mediante actividades sociales, empresariales o de innovación (García-Peñalvo, 2016).

De igual importancia, el emprendimiento ha sido considerado como un eje e impulsor por el cual las universidades dan cumplimiento de su tercera misión a través de la generación y apoyo a negocios, o bien, mediante la formación de sus estudiantes dotándoles de herramientas que fomenten la creación de empresas (Piirainen et al., 2016). La literatura en educación y emprendimiento ha consignado la importancia de que las universidades generen instancias de educación emprendedora que permitan a los estudiantes desarrollar actitudes frente al emprendimiento y los procesos de creación de negocios (Fayolle y Gailly, 2015). Lo anterior releva la importancia de estudiar está temática dado que, comprender como desde la formación se aporta a potenciar las competencias en emprendimiento de los estudiantes, resulta crucial en la instalación de la misión vinculante con el medio y el aporte al desarrollo local que éstos puedan impactar como futuros profesionales.

El presente estudio tiene como objetivo analizar los factores que conforman la actitud emprendedora de los universitarios de primer año utilizando una muestra de 322 estudiantes de la Universidad Católica del Maule, Chile, medida a través de una escala basada en el enfoque de Orientación a las Actitudes Emprendedoras propuesta por Robinson et al. (1991). Para ello, se utilizó análisis factorial exploratorio para lograr identificar las dimensiones que componen el perfil de actitudes emprendedoras de los nuevos estudiantes y técnicas de análisis inferencial en la comparación de grupos. Las conclusiones del estudio permiten orientar acciones en estudiantes universitarios, planteando la necesidad de realizar mediciones diagnósticas que permitan perfilar las estrategias considerando la diversidad de estudiantes que ingresan a la educación superior. Los siguientes apartados muestran los antecedentes teóricos, metodología, resultados, discusión, y finalmente, las conclusiones del estudio realizado.

El emprendimiento ha sido estudiado desde múltiples disciplinas, como por ejemplo la economía, psicología y sociología, entre otros. Sin embargo, ningún estudio es concluyente al respecto quedando bastante claro que es un tema complejo, multifactorial y que aborda determinantes tanto individuales, como socio-culturales (Fernández-Serrano y Liñán, 2014; Valliere, 2017). Las principales visiones con respecto a las dimensiones que componen la capacidad emprendedora de los individuos son las características individuales y motivacionales (van Hemmen et al., 2013). En efecto, de acuerdo a la literatura se puede vincular la decisión de emprender tanto con las actitudes, las motivaciones, los rasgos de personalidad, las habilidades personales y el contexto (García et al., 2005).

El principal enfoque teórico ha sido el propuesto por Ajzen (1991) quién desarrolló un modelo de conducta planificada que mide la intención que se tiene previo a un determinado comportamiento y que ha sido ampliamente utilizado en el estudio de la intención emprendedora en estudiantes. Caben consignar, por ejemplo: Fietze y Boyd (2017) en estudiantes daneses, Fragoso et al. (2020) en estudiantes brasileños y portugueses o Farhat y Guevara (2019) a estudiantes ecuatorianos. En general, este enfoque de conducta planificada ha considerado relevante los aspectos referidos a la actitud, normas sociales o subjetivas y la capacidad percibida (Ajzen, 1991). Un enfoque alternativo ha sido el desarrollado por Robinson et al. (1991) basado en modelo de Orientación a la Actitud Emprendedora (en adelante, OAE). La OAE se encuentra centrada en las actitudes, tomando como referencia que es el principal determinante de la acción de emprender. Es por esto que se diferencia de los modelos clásicos propuestos por Ajzen (1991) o Krueger y Brazeal (1994), que si bien tienen elementos comunes con Robinson et al. (1991) en cuanto a la capacidad percibida y el control interno, este último se centra en como las actitudes y los rasgos de personalidad impactan en el comportamiento emprendedor.

En consideración a lo anterior, de acuerdo a autores como Jena (2020) las actitudes pueden entenderse como la inclinación positiva, negativa o neutral de una persona por un objeto o idea. Es por esto que, en el contexto del emprendimiento las actitudes pueden vincularse a las intenciones por emprender, tomando en consideración que reflejan una medida de como un individuo percibe el comportamiento empresarial y sus consecuencias (Ajzen, 1991). Así, las actitudes por el emprendimiento pasan a ser una medida de la intención por emprender en los individuos permitiendo capturar la actitud general hacia el comportamiento empresarial. Referente a las actitudes emprendedoras, si bien han sido consideradas por la literatura recientemente, su desarrollo es aún incipiente por lo que los estudios desarrollados en esta línea son un aporte a la comprensión de la relación entre actitudes e intención por emprender. Autores como Wardana et al. (2020) señalan la 
relación directa y significativa entre las actitudes emprendedoras y la mentalidad por emprender, por ende, son consideradas como una dimensión relevante de la intención por emprender futura por parte los estudiantes. Este estudio hace uso de actitudes por emprender de los estudiantes, considerando que son una medida de la intención y, por tanto, del potencial por emprender considerando que el modelo OAE propone que las actitudes se presentan como el mejor enfoque para la descripción de los emprendedores (Robinson et al., 1991).

El modelo OAE ha sido utilizado frecuentemente para analizar la intención emprendedora de los estudiantes universitarios (por ejemplo: Jena, 2020; Krauss, 2011). Esta teoría considera que la acción por emprender esta mediada por actitudes referidas a: la necesidad de logro, la innovación, el control interno y la autoestima (Robinson et al., 1991). De acuerdo a este autor, las actitudes y habilidades que interfieren en el proceso emprendedor condicionan el éxito en la conducta empresarial. Con respecto a lo anterior, la necesidad de logro es identificada por Robinson et al. (1991) como el planteamiento de metas y las acciones tendientes a lograrla. En tanto, el control percibido interno se refiere la percepción que tiene un individuo sobre su capacidad para realizar la tarea, lo que es concordante con la TCP de Ajzen (1991). Referente a la innovación Robinson et al. (1991) lo define como la búsqueda nuevas oportunidades y la capacidad para enfrentarlas, donde Krauss (2011) además considera la gestión de riesgos. En relación a la autoestima, se identifica como la seguridad en sí mismo y la confianza con la cual un individuo enfrenta la capacidad por emprender (Robinson et al., 1991).

En consideración a los factores determinantes de la decisión de emprender, se han considerado moderadores atribuibles a características personales, psicológicas y otros antecedentes sociodemográficos. En este contexto, Herman (2019) analizó que los rasgos de personalidad emprendedora influyen directamente en el potencial de un estudiante y en la decisión de emprender. Por otro lado, estudios como el de Anggadwita et al. (2017) han comprobado el efecto de variables como el ambiente sociocultural y de género sobre la intención emprendedora. También, Abu Bakar et al. (2017) estudió los factores que determinan el emprendimiento identificando como variables relevantes a: el género, la educación, el ingreso, el miedo al fracaso y conocimiento de otros empresarios.

En contraparte, algunos autores han identificado que el ingreso familiar o la existencia de familiares emprendedores impactan favorablemente la intención por emprender (Gujrati et al., 2019). El estudio tradicional de la intención por emprender también ha analizado como un determinante el nivel de instrucción y educación, aludiendo a que genera una mayor capacidad de innovación y desarrollo individual, lo que releva la importancia de las universidades (Fayolle y Gailly, 2015). Dentro del contexto universitario, existen varios estudios que apoyan que la educación modifica positivamente la intención emprendedora, por lo que las universidades pueden funcionar como un instrumento que potencia la actividad empresarial (Fragoso et al., 2020; Hendrajaya et al., 2019).

En Chile se han realizado algunos estudios sobre la intención por emprender de estudiantes de educación superior, tal como Soria-Barreto et al. (2016). Las principales orientaciones metodológicas son la aplicación de cuestionarios de valoración y percepción de los estudiantes. Igualmente, Bretones y Radrigán (2018) ha incluido en la discusión la variable cultural, aludiendo a las diferencias entre estudiantes de contextos diversos. Este estudio amplía esta discusión, incluyendo la necesidad de perfilar un diagnóstico del potencial emprendedor y desde aquí generar estrategias que permitan potenciar las habilidades de los estudiantes a partir de la teoría enfocada en la Orientación de Actitudes Emprendedoras. En este contexto, la contribución de este trabajo está enfocada en el estudio de intención por emprender medida por las actitudes empresariales de los estudiantes, situación menos abordada en literatura nacional e internacional y que permite una explicación de los factores que interfieren en que, eventualmente los estudiantes tengan actitudes referentes a la creación de empresas. Lo anterior, en un contexto en que las universidades son en sí un ambiente positivo para la capacitación empresarial de los estudiantes mediante el conocimiento, las ideas y el aprendizaje (Alves et al., 2019).

\section{METODOLOGÍA}

Este estudio tiene un enfoque cuantitativo de tipo descriptivo siendo su objetivo principal analizar los factores que conforman la actitud emprendedora de los universitarios de primer año. Los objetivos específicos de la investigación fueron: 1) Determinar las dimensiones de la actitud emprendedora en los estudiantes de primer año; y 2) Analizar diferencias en las actitudes e intención emprendedora de los estudiantes por áreas de estudio, género, percepción de trabajo ideal y capacidad percibida. Se utilizaron datos de corte transversal a través de la aplicación de una encuesta a estudiantes universitarios de primer año de las áreas de Ingeniería y Administración y Economía de la Universidad Católica del Maule, Chile. En los siguientes apartados se exponen en detalle la muestra, el instrumento de medición utilizado y metodología utilizada para el procesamiento de los datos. 


\section{Participantes y muestra}

Los participantes corresponden a estudiantes de primer año de la Universidad Católica del Maule, Chile. Se utilizó un muestreo no probabilístico por conveniencia de estudiantes que cursaban primer año en carreras de las áreas de Ingeniería y Administración y Economía, de la sede Talca. La participación de los estudiantes fue realizada de manera anónima, voluntaria y exentos de cualquier pago asociado. El instrumento fue aplicado a 322 individuos mediante un cuestionario tipo auto reporte. La Tabla 1 resume las variables utilizadas en el estudio. Cabe mencionar que un $67,7 \%$ de los estudiantes en la muestra son hombres, mientras que un $32,3 \%$ son mujeres. Con respecto a la carrera que cursan, se puede apreciar una distribución no homogénea con grupos que oscilan entre 66 y 35 participantes. Referente a la actividad laboral, los encuestados señalan en un $74,8 \%$ no realizar actividad laboral mientras estudian frente al $25,2 \%$ que si lo realiza. En relación al trabajo ideal, un $49,1 \%$ indica que tener una empresa propia es su ideal, mientras que un $23,6 \%$ señala que tener una empresa en equipo. Finalmente, un $28,3 \%$ señala tener las capacidades necesarias desde lo teórico y un $25,8 \%$ señala necesitar de otras personas para emprender. El detalle para todas las categorías puede observarse en la Tabla 1.

Tabla 1: Caracterización de la muestra

\begin{tabular}{|c|c|c|c|c|c|c|c|}
\hline \multicolumn{2}{|r|}{ Variable } & $N$ & Porc. & \multicolumn{2}{|r|}{ Variable } & $N$ & Porc. \\
\hline Género & $\begin{array}{l}\text { Hombre } \\
\text { Mujer }\end{array}$ & $\begin{array}{l}218 \\
104\end{array}$ & $\begin{array}{l}67,7 \% \\
32,3 \%\end{array}$ & $\begin{array}{l}\text { Actividad } \\
\text { Laboral }\end{array}$ & $\begin{array}{l}\mathrm{Si} \\
\text { No }\end{array}$ & $\begin{array}{c}81 \\
241\end{array}$ & $\begin{array}{l}25,2 \% \\
74,8 \%\end{array}$ \\
\hline $\begin{array}{c}\text { Carrera } \\
\text { que cursa }\end{array}$ & $\begin{array}{l}\text { Ing. Comercial } \\
\text { Ing. Civil } \\
\text { Ing. Civil Industrial } \\
\text { Ing. Civil Informática } \\
\text { Ing. En Construcción } \\
\text { Contador Auditor }\end{array}$ & $\begin{array}{l}54 \\
63 \\
35 \\
58 \\
66 \\
46\end{array}$ & $\begin{array}{l}16,8 \% \\
19,6 \% \\
10,9 \% \\
18,0 \% \\
20,5 \% \\
14,3 \%\end{array}$ & $\begin{array}{l}\text { Trabajo } \\
\text { Ideal }\end{array}$ & $\begin{array}{l}\text { Funcionario público } \\
\text { Empresa propia } \\
\text { Empresa en equipo } \\
\text { Asalariado gran empresa } \\
\text { Asalariado pequeña empresa } \\
\text { Alto directivo } \\
\text { Soy pesimista de ser } \\
\text { emprendedor } \\
\text { Otro }\end{array}$ & $\begin{array}{c}19 \\
158 \\
76 \\
26 \\
4 \\
33 \\
1 \\
5\end{array}$ & $\begin{array}{c}5,9 \% \\
49,1 \% \\
23,6 \% \\
8,1 \% \\
1,2 \% \\
10,2 \% \\
0,3 \% \\
1,6 \%\end{array}$ \\
\hline & & & & $\begin{array}{l}\text { Capacidad } \\
\text { para } \\
\text { emprender }\end{array}$ & $\begin{array}{l}\text { No tengo capacidad } \\
\text { Escasas } \\
\text { Tengo lo necesario (teórico) } \\
\text { Tengo lo necesario (práctico) } \\
\text { Soy capaz } \\
\text { No se mi capacidad } \\
\text { No necesita capacidad } \\
\text { Necesito otras personas }\end{array}$ & $\begin{array}{c}2 \\
33 \\
91 \\
41 \\
38 \\
29 \\
5 \\
83 \\
\end{array}$ & $\begin{array}{r}0,6 \% \\
10,2 \% \\
28,3 \% \\
12,7 \% \\
11,8 \% \\
9,0 \% \\
1,6 \% \\
25,8 \% \\
\end{array}$ \\
\hline
\end{tabular}

\section{Instrumento de medición}

El instrumento corresponde al aplicado por Krauss (2011) en estudios que miden el potencial emprendedor de estudiantes de educación superior. Este empleó una escala enfocada en la teoría de Orientación a la Actitud Emprendedora propuesta por Robinson et al. (1991). El enfoque OAE e instrumentos asociados han sido utilizados por diversos autores en el contexto universitario obteniendo buenos indicadores de consistencia interna y fiabilidad (por ejemplo: Jena, 2020; Krauss, 2011). El constructo se encuentra basado en la teoría de OAE que tiene enfoque en las actitudes. Por lo anterior, la escala es útil en la identificación de perfiles de emprendedores entre los estudiantes universitarios permitiendo orientar diagnósticos y estrategias a través de la educación en emprendimiento. El enfoque de Robinson et al. (1991) considera que la actitud se presenta como el mejor moderador de la intención por emprender y se encuentra basado en cuatro dimensiones: logro, control personal, innovación y autoestima. Los participantes respondieron a la escala de 30 afirmaciones respecto de su nivel de acuerdo (escala Likert de seis niveles). Del total de ítems, en el procesamiento se seleccionaron 24 reactivos para salvaguardar su peso factorial en las dimensiones. Se evaluó la consistencia interna del instrumento a través de distintos indicadores de confiabilidad obteniendo resultados apropiados (Alfa de Cronbach de 0,85; Dos mitades de Guttman de 0,85; y Omega de 0,89). Además, se incluyó la consulta de datos sociodemográficos como género, carrera que cursa, percepción de capacidad y sus intenciones emprendedoras futuras.

\section{Procesamiento de los datos}

Las encuestas fueron aplicadas como un auto reporte, posteriormente tabuladas en planillas de cálculo y validadas en términos de consistencia con las respuestas de los cuestionarios. El procesamiento estadístico se realizó mediante el software Rstudio. En el análisis, se realizaron análisis descriptivos de la muestra (ver Tabla 1). Posteriormente, se utilizó la técnica de análisis factorial exploratorio (AFE) con la finalidad de reducir dimensiones y determinar los ítems para conformar los constructos de las actitudes emprendedoras de 
acuerdo con el modelo OAE que interfieren en la intención emprendedora de los estudiantes de primer año. Una de las principales ventajas de esta técnica es que permite identificar apropiadamente los factores subyacentes a través de los ítems en la escala de medición, lo que es apropiado tomando en consideración el objetivo del estudio. No obstante, algunas desventajas son la dificultad de elegir el método apropiado de estimación e interpretar el significado de los factores, lo que en este caso es subsanado tomando en consideración que la base de los factores se encuentra supeditada al modelo OAE.

Tomando en consideración lo anteriormente expuesto, en el AFE se utilizó el método de mínimos cuadrados ponderados con solución rotada "varimax", considerando la naturaleza de las respuestas de los ítems (escala Likert de seis niveles). Se realizó primero un pre-procesamiento de la escala original de 30 afirmaciones, notando que no cumplía con que todos los ítems tuvieran una comunalidad superior a 0,30. Fueron eliminados los ítems que no cumplieran con este requisito y finalmente, se procesó la escala con 24 reactivos. Del proceso de extracción se obtuvieron cuatro dimensiones concordantes con la literatura y el modelo OAE. Para su posterior procesamiento, se obtuvo la puntuación de cada factor y se ponderaron de acuerdo a la varianza explicada obteniendo la variable "intención emprendedora global" (en adelante, IEG) que representa una medida del grado de intención por emprender en los estudiantes. Con el propósito de analizar la existencia de diferencias entre los factores identificados, la intención emprendedora global y las características demográficas de los estudiantes, se utilizaron estadísticos inferenciales. Se analizó la normalidad de las variables mediante las pruebas Lilliefors y Shapiro-Willks. A la postre, se utilizaron pruebas no paramétricas considerando la no normalidad basando los análisis en las pruebas de Wilcoxon y Kruskall-Wallis. Una ventaja de estas técnicas no paramétricas es que hacen menos suposiciones pudiendo ser implementadas en muestras que son menos balanceadas entre grupos y que no poseen normalidad como es expuesto en este estudio.

\section{RESULTADOS}

Se muestran primero los resultados del AFE realizado y posteriormente, los resultados a nivel inferencial tomando como base las dimensiones encontradas.

\section{Resultados AFE}

Referente al análisis factorial exploratorio (AFE), se analizó en primera instancia la factibilidad mediante las pruebas de Kaiser-Meyer-Olkin $(\mathrm{KMO}=0,820)$ y la prueba de esfericidad de Bartlett (estadístico $\mathrm{X}^{2}(210)=$ 1.531,946; $p$-valor < 0,001) indicando que es apropiado el análisis. En la selección de las dimensiones se consideraron valores propios superiores a 1,0 (ver Figura 1), lo que permitió identificar cuatro factores que explican un $49 \%$ de la varianza total. La Tabla 2 muestra las cargas factoriales obtenidas mediante el método de mínimos cuadrados ponderados y rotación ortogonal "varimax" para cada dimensión. Los cuatro factores fueron denominados de acuerdo a la literatura y la teoría de OAE: 1) Factor 1 - Autoestima; 2) Factor 2 Control personal o interno; 3) Factor 3 - Asunción de riesgo e innovación; y 4) Factor 4 - Necesidad de logro.

A partir de los resultados del AFE, se construyó la variable "intención emprendedora global" (IEG) en virtud de la puntuación obtenida por los estudiantes en cada dimensión ponderada por su aporte a la varianza total. Las estadísticas descriptivas y pruebas de normalidad para las dimensiones y la IEG se muestran en la Tabla 3 (niveles de significancia: ** $1 \%$ y * $5 \%$; M representa la media, DE la desviación estándar y ME la mediana). También, en la Tabla 3 se puede apreciar el "Logro Medio" obtenido desde el puntaje total por cada dimensión sobre la Media (M). Se puede apreciar un "Logro Medio" de la IEG en torno al $82 \%$, donde la dimensión más baja es la relacionada con el control interno $(65,06 \%)$ y la mayor está asociada a la necesidad de logro $(84,59 \%)$.

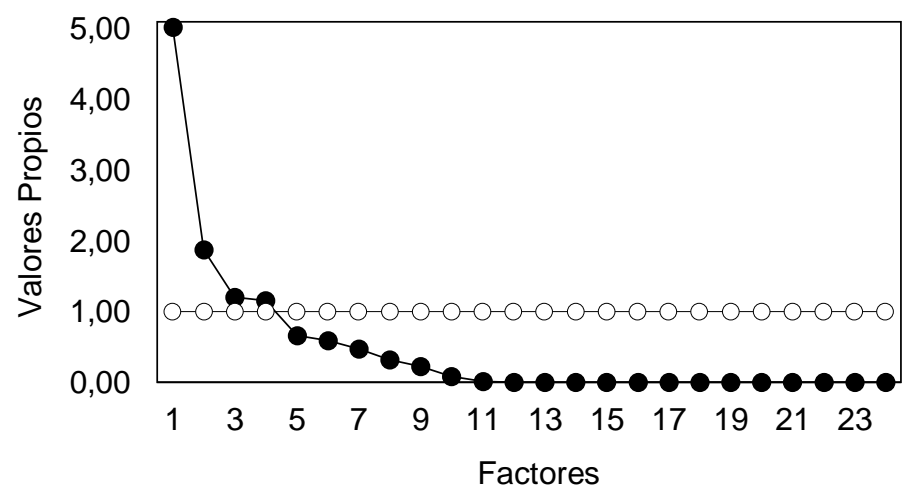

Fig. 1: Gráfica de sedimentación 
Tabla 2: Cargas factoriales

\begin{tabular}{|c|c|c|c|c|c|}
\hline \multirow[b]{2}{*}{ Afirmación } & \multirow[b]{2}{*}{ Comunalidades } & \multicolumn{4}{|c|}{ Factores } \\
\hline & & Autoestima & $\begin{array}{c}\text { Control personal } \\
\text { o interno }\end{array}$ & $\begin{array}{c}\text { Asunción de riesgo } \\
\text { e innovación }\end{array}$ & $\begin{array}{l}\text { Necesidad } \\
\text { de logro }\end{array}$ \\
\hline Pregunta 9 & 0,72 & 0,81 & & & \\
\hline Pregunta 15 & 0,40 & 0,59 & & & \\
\hline Pregunta 25 & 0,45 & 0,58 & & & \\
\hline Pregunta 29 & 0,55 & 0,58 & & & \\
\hline Pregunta 20 & 0,44 & 0,57 & & & \\
\hline Pregunta 24 & 0,42 & 0,56 & & & \\
\hline Pregunta 4 & 0,40 & 0,45 & & & \\
\hline Pregunta 6 & 0,35 & 0,40 & & & \\
\hline Pregunta 17 & 0,61 & & & & 0,70 \\
\hline Pregunta 19 & 0,50 & & & & 0,70 \\
\hline Pregunta 7 & 0,63 & & & & 0,65 \\
\hline Pregunta 2 & 0,31 & & & & 0,47 \\
\hline Pregunta 28 & 0,35 & & & & 0,47 \\
\hline Pregunta 16 & 0,35 & & & & 0,44 \\
\hline Pregunta 10 & 0,40 & & & & 0,41 \\
\hline Pregunta 8 & 0,57 & & 0,71 & & \\
\hline Pregunta 11 & 0,71 & & 0,69 & & \\
\hline Pregunta 27 & 0,50 & & 0,66 & & \\
\hline Pregunta 13 & 0,48 & & 0,64 & & \\
\hline Pregunta 1 & 0,46 & & 0,49 & & \\
\hline Pregunta 14 & 0,43 & & 0,48 & & \\
\hline Pregunta 23 & 0,31 & & & 0,37 & \\
\hline Pregunta 30 & 0,83 & & & 0,89 & \\
\hline Pregunta 18 & 0,52 & & & 0,43 & \\
\hline \multicolumn{2}{|c|}{ Valores propios } & 5,03 & 1,88 & 1,21 & 1,16 \\
\hline \multicolumn{2}{|c|}{ Varianza explicada del factor } & 0,15 & 0,13 & 0,08 & 0,13 \\
\hline \multicolumn{2}{|c|}{ Varianza explicada acumulada } & 0,15 & 0,28 & 0,36 & 0,49 \\
\hline
\end{tabular}

Tabla 3: Descriptivas principales

\begin{tabular}{|c|c|c|c|c|c|c|c|c|c|}
\hline \multirow[b]{2}{*}{ Factores } & \multirow[b]{2}{*}{$N$} & \multirow[b]{2}{*}{$M$} & \multirow[b]{2}{*}{$D E$} & \multirow[b]{2}{*}{$M E$} & \multirow{2}{*}{$\begin{array}{c}N \\
\text { Ítems }\end{array}$} & \multirow{2}{*}{$\begin{array}{l}\text { Logro } \\
\text { Medio }\end{array}$} & \multicolumn{2}{|c|}{ Normalidad } & \multirow{2}{*}{$\begin{array}{c}\text { Conclusió } \\
n\end{array}$} \\
\hline & & & & & & & Lilliefors & $\begin{array}{l}\text { Shapiro } \\
\text {-Willks }\end{array}$ & \\
\hline Autoestima & 322 & 39,72 & 3,86 & 40 & 8 & $82,75 \%$ & $0,08^{* *}$ & $0,98^{* *}$ & No normal \\
\hline $\begin{array}{l}\text { Control personal o } \\
\text { interno }\end{array}$ & 322 & 23,42 & 4,97 & 23 & 6 & $65,06 \%$ & $0,07^{* *}$ & $0,99^{*}$ & No normal \\
\hline $\begin{array}{l}\text { Asunción de riesgo e } \\
\text { innovación }\end{array}$ & 322 & 13,01 & 2,43 & 13 & 3 & $72,28 \%$ & $0,12^{* *}$ & $0,96^{* *}$ & No normal \\
\hline Necesidad de logro & 322 & 35,53 & 3,53 & 36 & 7 & $84,59 \%$ & $0,09^{* *}$ & $0,95^{\star *}$ & No normal \\
\hline IEG & 322 & 29,92 & 2,90 & 29,91 & 24 & $81,99 \%$ & $0,05^{*}$ & $0,99^{*}$ & No normal \\
\hline
\end{tabular}

\section{Resultados inferenciales}

Dada la naturaleza no normal de las variables (ver Tabla 3) se utilizaron pruebas no paramétricas en el análisis inferencial. Se utilizaron las pruebas de Wilcoxon (dos grupos) y Kruskall-Wallis (más de dos grupos) tomando como referencia la comparación de medianas (ME) con un nivel de significancia del 95\%. En la Tabla 4 se pueden apreciar las diferencias por disciplina. En esta se observan diferencias a favor de los estudiantes del área de Administración y Economía respecto del factor Autoestima $(\mathrm{W}=9.198$; $p$-valor $<0,050$; t.e. $=0,14)$ y la IEG $(W=9.331$; p-valor <0,050; t.e. $=0,13)$. En la Tabla 5 se pueden apreciar las diferencias por género. En esta se observan diferencias a favor de los hombres respecto del factor Control personal o interno $(\mathrm{W}=$ 13.980; $p$-valor $<0,001$; t.e. $=0,19)$. También, se observan diferencias significativas a favor de las mujeres para la dimensión Necesidad de logro $(\mathrm{W}=9.632$; $p$-valor $<0,050$; t.e. $=0,12)$. Cabe mencionar que no se apreciaron diferencias significativas por género en torno a la IEG. 
Tabla 4: Diferencias por área carrera - prueba Wilcoxon

\begin{tabular}{|c|c|c|c|c|c|c|c|}
\hline \multirow{2}{*}{ Factores } & \multicolumn{3}{|c|}{$\begin{array}{l}\text { Administración y Economía } \\
\qquad(n=100)\end{array}$} & \multicolumn{3}{|c|}{ Ingeniería (n=222) } & \multirow{2}{*}{ Diferencia } \\
\hline & $M$ & $D E$ & $M E$ & $M$ & $D E$ & $M E$ & \\
\hline Autoestima & 40,56 & 3,69 & 41 & 39,34 & 3,89 & 39 & Sig. \\
\hline Control personal o interno & 24,08 & 5,11 & 24 & 23,13 & 4,89 & 23 & No sig. \\
\hline Asunción de riesgo e innovación & 13,08 & 2,24 & 13 & 12,97 & 2,51 & 13 & No sig. \\
\hline Necesidad de logro & 36,11 & 3,18 & 36 & 35,27 & 3,66 & 35 & No sig. \\
\hline IEG & 30,52 & 2,81 & 30,14 & 29,65 & 2,91 & 29,6 & Sig. \\
\hline
\end{tabular}

Tabla 5: Diferencias por género - prueba Wilcoxon

\begin{tabular}{|l|c|c|c|c|c|c|c|}
\hline \multirow{2}{*}{ Factores } & \multicolumn{3}{c|}{ Hombre $(n=218)$} & \multicolumn{3}{c|}{ Mujer $(n=104)$} & \multirow{2}{*}{ Diferencia } \\
\cline { 2 - 7 } & $M$ & $D E$ & $M E$ & $M$ & $D E$ & ME & \\
\hline Autoestima & 39,71 & 4,04 & 40 & 39,74 & 3,47 & 40 & No sig. \\
\hline Control personal o interno & 24,06 & 4,88 & 24 & 22,08 & 4,92 & 22 & Sig. \\
\hline Asunción de riesgo e innovación & 13,12 & 2,38 & 13 & 12,76 & 2,51 & 13 & No sig. \\
\hline Necesidad de logro & 35,17 & 3,73 & 35 & 36,28 & 2,95 & 36 & Sig. \\
\hline IEG & 30,01 & 3 & 30,5 & 29,73 & 2,7 & 29,44 & No sig. \\
\hline
\end{tabular}

En la Tabla 6, se pueden observar las diferencias por trabajo ideal considerando a aquellos estudiantes que señalaron como idóneo el trabajar como emprendedor, contra los que de preferencia piensan ser directivos, funcionarios o asalariados. En este caso, se han identificado diferencias significativas a favor de quienes se perciben como futuros emprendedores en las dimensiones de Autoestima $(\mathrm{W}=8.738$; $p$-valor < 0,050; t.e. = 0,12); Control personal o interno $(\mathrm{W}=8.464$; $p$-valor < 0,050; t.e. = 0,14); Asunción de riesgo e innovación (W $=7.967$; p-valor < 0,001; t.e. =0,18); y también, sobre la IEG $(\mathrm{W}=8.144$; $\mathrm{p}$-valor $<0,001$; t.e. $=0,16)$. No se observaron diferencias para el factor necesidad de logro.

Tabla 6: Diferencias por trabajo ideal - prueba Wilcoxon

\begin{tabular}{|l|c|c|c|c|c|c|c|}
\hline \multirow{2}{*}{ Factores } & \multicolumn{3}{c|}{ No emprendedor $(n=88)$} & \multicolumn{3}{c|}{ Emprendedor $(n=234)$} & \multirow{2}{*}{ Diferencia } \\
\cline { 2 - 7 } & $M$ & $D E$ & $M E$ & $M$ & $D E$ & $M E$ & \\
\hline Autoestima & 38,8 & 4,4 & 39 & 40,06 & 3,59 & 40 & Sig. \\
\hline Control personal o interno & 22,26 & 4,54 & 23 & 23,86 & 5,06 & 24 & Sig. \\
\hline Asunción de riesgo e innovación & 12,33 & 2,37 & 12 & 13,26 & 2,41 & 13 & Sig. \\
\hline Necesidad de logro & 35,12 & 3,92 & 35 & 35,68 & 3,37 & 36 & No sig. \\
\hline IEG & 29,11 & 2,89 & 29,21 & 30,23 & 2,85 & 30,1 & Sig. \\
\hline
\end{tabular}

Respecto de la capacidad percibida (Tabla 7), se puede evidenciar diferencias significativas en todos los niveles (baja, media y alta capacidad percibida) respecto de los factores. Esta última se construyó a partir de los niveles bajo (no tengo capacidad, escasas y no se mi capacidad), medio (tengo lo necesario (teórico y práctico) y necesito otras personas) y alto (soy capaz y no necesita capacidad). Así, se observaron diferencias en las dimensiones asociados a Autoestima $\left(X^{2}(2)=22,42\right.$; p-valor < 0,001; t.e. $\left.=0,06\right)$; Control personal o interno $\left(x^{2}(2)=20,53\right.$; p-valor < 0,001; t.e. = 0,06); Asunción de riesgo e innovación $\left(x^{2}(2)=10,01 ; p\right.$-valor $<$ $0,001$; t.e. $=0,03)$; Necesidad de logro $\left(x^{2}(2)=12,29\right.$; p-valor < 0,001; t.e. $\left.=0,03\right)$; y la IEG $\left(x^{2}(2)=31,69\right.$; $p$ valor $<0,001$; t.e. $=0,09)$.

Tabla 7: Diferencias por capacidad percibida - prueba Kruskall-Wallis

\begin{tabular}{|l|c|c|c|c|c|c|c|c|c|c|}
\hline \multirow{2}{*}{ Factores } & \multicolumn{3}{c|}{ Baja $(n=64)$} & \multicolumn{3}{c|}{ Media $(n=215)$} & \multicolumn{3}{c|}{ Alta $(n=43)$} & Diferenc \\
ia & \\
\cline { 2 - 11 } & $M$ & $D E$ & $M E$ & $M$ & $D E$ & $M E$ & $M$ & $D E$ & $M E$ & Sig. \\
\hline Autoestima & 37,8 & 4,07 & 38 & 39,93 & 3,6 & 40 & 41,49 & 3,74 & 42 & Sig. \\
\hline Control personal o interno & 20,91 & 4,92 & 21 & 23,8 & 4,81 & 24 & 25,26 & 4,58 & 25 & Sig. \\
\hline Asunción de riesgo e innovación & 12,33 & 2,38 & 12,5 & 13,05 & 2,42 & 13 & 13,79 & 2,32 & 14 & Sig. \\
\hline Necesidad de logro & 33,81 & 4,61 & 34 & 35,91 & 3,08 & 36 & 36,19 & 3,05 & 36 & Sig \\
\hline IEG & 28,1 & 2,89 & 28 & 30,2 & 2,72 & 30,12 & 31,25 & 2,61 & 31,2 & Sig. \\
\hline
\end{tabular}




\section{DISCUSIÓN}

El estudio del emprendimiento y sus determinantes componen una cantidad múltiple de factores interrelacionados (Valliere, 2017). Este estudio logró identificar cuatro dimensiones que explican las actitudes emprendedoras de los estudiantes universitarios de primer año, las que son concordantes con la teoría de Orientación a las Actitudes Emprendedora (Robinson et al., 1991). Estos factores se encuentran asociados a: 1) Autoestima; 2) Control personal o interno; 3) Asunción de riesgo e innovación; y 4) Necesidad de logro. Este enfoque es considerado por Robinson et al. (1991) como un modelo apropiado para la explicación de la conducta emprendedora, por lo cual contribuye en la medición de las intenciones por emprender desde las actitudes de los individuos. Por lo anterior, ofrece una medida interesante para modelar la intención futura en emprendimiento considerando que autores como Jena (2020) o Wardana et al. (2020) han encontrado relación directa con las actitudes de los estudiantes.

Tras los resultados, se logró apreciar algunas diferencias entre las áreas disciplinares a favor de aquellas vinculadas a la Administración y Economía frente a las de Ingeniería. Estas diferencias, se observaron en el factor Autoestima que según Robinson et al. (1991) refiere a la confianza en sí mismo por enfrentar el proceso emprendedor. Lo anterior, hace mención a las diferencias entre los perfiles de ingreso de los estudiantes de las carreras, donde los estudiantes de Administración se encuentran mayormente perfilados con el emprendimiento. Las diferencias en los perfiles de ingreso de los estudiantes y sus expectativas profesionales han sido relevadas en estudios como Fietze y Boyd (2017). También, en términos de intención emprendedora global (IEG) se puede observar diferencias significativas entre disciplinas.

En relación a la variable género, no se lograron evidenciar diferencias significativas entre los grupos de hombres y mujeres respecto de la IEG, tal como en el caso de Soria-Barreto et al. (2016) para estudiantes universitarios chilenos. En la literatura internacional, el estudio realizado por Baker y Welter (2017) intenta erradicar la idea de las diferencias de género en los riesgos tomados en el emprendimiento, concluyendo que, es el contexto socioeconómico y cultural el que determina el riesgo asumido por el emprendedor. No obstante, estudios de intención emprendedora en estudiantes han encontrado diferencias entre hombres y mujeres (Abu Bakar et al., 2017). En este caso, si bien no se evidencia diferencia en IEG, si se encuentran en algunos factores asociados a la actitud por emprender. Por ejemplo, se evidenciaron diferencias a favor de los hombres para la dimensión Control personal o interno, y a favor de las mujeres en la dimensión Necesidad de logro.

Referente a la percepción de capacidad emprendedora, se denotan diferencias significativas entre los grupos que se perciben altamente capaces de enfrentar la acción de emprender y quienes muestran un menor grado de capacidad percibida. Este resultado es evidenciable en el caso chileno en Soria-Barreto et al. (2016) y también, a nivel internacional para los estudios bajo la TCP (por ejemplo: Farhat y Guevara, 2019; Fragoso et al., 2020). Del mismo modo, estudios como el realizado por Ahmed et al., (2017) han señalado la existencia de diferencias significativas entre la intención emprendedora y la capacidad percibida de los estudiantes. La identificación de diferencias significativas entre los grupos de estudiantes que se perciben capaces o no de emprender al futuro, descubre la necesidad de generar estrategias que permitan a los estudiantes desarrollar capacidades en entornos simulados y reales en los planes de estudios, siendo esta componente altamente interesante de analizar desde la perspectiva práctica de la formación profesional en emprendimiento.

Dentro de la misma línea, McCann (2017) ha señalado que el emprendimiento tiene relación con las expectativas futuras de los estudiantes. Justamente, quienes perciben tener una capacidad superior en torno a la conformación de negocios, poseen una IEG superior a los estudiantes que no creen ser capaces de emprender. En vinculación con lo anterior, el presente estudio también permitió observar diferencias significativas entre los estudiantes que perciben como trabajo ideal emprender su propio negocio, en desmedro de aquellos grupos que prefieren ser empleados por otros. Este último resultado es equivalente al obtenido por Fietze y Boyd (2017) lo que permite comprobar que, para el caso de los estudiantes universitarios chilenos, la intención de emprender futura como trabajo ideal es un atenuante de las actitudes e intención por emprender. Lo anterior, además es concordante con autores como Wardana et al. (2020) quienes señalan que los estudiantes elegirán una carrera como emprendedor porque se sienten satisfechos de las oportunidades que esta condición les ofrece.

En resumen, se encontraron diferencias por disciplina lo que sugiere estrategias específicas para cada una de estas. Estas diferencias son producidas principalmente en la dimensión de Autoestima, referida a la confianza en sí mismo y la visualización de acciones para enfrentar el futuro como emprendedor. Lo anterior está mediado por el perfil de ingreso de los estudiantes y su motivación por el área disciplinar. En consecuencia, se releva la necesidad de generar diagnósticos particulares para las diferentes carreras antes de generar planes genéricos referidos a potenciar las competencias y actitudes emprendedoras de los estudiantes. Del mismo modo y de acuerdo a los resultados, los estudiantes de primer año identifican como factores relevantes la percepción de ser empresario como trabajo ideal y la capacidad para lograrlo. Ambas favorecen la intención emprendedora, por ende, se sugiere utilizar estrategias metodológicas y de aprendizaje 
enfocadas en casos prácticos o prácticas tempranas, vinculadas con el medio emprendedor. Esto sugiere que los estudiantes se enfrenten a conocer la actividad empresarial y mejorar su percepción respecto de la capacidad propia.

Esta investigación proporciona elementos útiles a la hora de definir estrategias para potenciar el emprendimiento dentro de los estudiantes, considerando que la educación permite desarrollar las habilidades, actitudes y competencias emprendedoras (Hendrajaya et al., 2019). De la misma forma, se puede citar el trabajo propuesto por Fayolle y Gailly (2015), quienes aluden que la intención de emprender está delimitada por variables demográficas, la capacidad percibida y finalmente, a la educación emprendedora. Esto sugiere la importancia de las universidades en la generación de capacidades en sus estudiantes respecto del potencial emprendedor, la vinculación con el medio emprendedor y su compromiso con la consecución de la tercera misión por esta vía. Como limitación puede considerarse el haber trabajado una muestra intencionada de estudiantes universitarios de primer año de las áreas de Economía y Negocios e Ingeniería en un centro poblacional específico. Lo anterior, puede llevar a limitaciones referidas a la homogeneidad de los participantes y hace necesario extender el estudio a grupos más heterogéneos de participantes, considerando estudiantes de diferentes disciplinas, niveles y centros poblacionales. Como líneas de trabajo futuro, se considera relevante el estudio de los factores determinantes de la actitud e intención emprendedora referidos a la capacidad percibida o autoeficacia empresarial de los estudiantes, notando que en este estudio se encontraron diferencias significativas de los grupos con bajo y alto grado de percepción sobre su propia capacidad de emprender.

\section{CONCLUSIONES}

De acuerdo a los resultados del estudio y la discusión realizada, se pueden resumir las siguientes conclusiones: 1) las dimensiones que componen la orientación actitudinal por el emprendimiento en universitarios de primer año chilenos corresponden a las referidas a autoestima, control personal o interno, asunción de riesgo e innovación, y necesidad de logro, concordantes a la teoría; y 2) la capacidad percibida por el estudiante y su percepción del emprendimiento como trabajo ideal, son variables relevantes de la actitud emprendedora evidenciada por los estudiantes. Estas dimensiones deben ser la base de las estrategias desarrolladas para potenciar la intención emprendedora de los estudiantes universitarios, basados en estrategias prácticas que permitan desarrollar sus habilidades y competencias durante su proceso formativo.

\section{REFERENCIAS}

Abu Bakar, A. R., Ahmad, S. Z., Wright, N. S., y Skoko, H., The propensity to business startup: evidence from global entrepreneurship monitor (gem) data in Saudi Arabia, https://doi.org/10.1108/JEEE-11-2016-0049, Journal of Entrepreneurship in Emerging Economies, 9(3), 263-285 (2017).

Ahmed, T., Chandran, V. G. R., y Klobas, J., Specialized entrepreneurship education: does it really matter? fresh evidence from Pakistan, https://doi.org/10.1108//JEBR-01-2016-0005, International Journal of Entrepreneurial Behavior \& Research, 23(1), 4-19 (2017).

Ajzen, I., The theory of planned behavior, https://doi.org/10.1016/0749-5978(91)90020-T, Organizational Behavior and Human Decision Processes, 50(2), 179-211 (1991).

Alves, A. C., Fischer, B., Schaeffer, P. R., y Queiroz, S., Determinants of student entrepreneurship: an assessment on higher education institutions in Brazil, https://doi.org/10.1108/INMR-02-2018-0002, Innovation \& Management Review, 16(2), 96-117 (2019).

Anggadwita, G., Luturlean, B. S., Ramadani, V., y Ratten, V., Socio-cultural environments and emerging economy entrepreneurship: women entrepreneurs in Indonesia, https://doi.org/10.1108/JEEE-03-2016-0011, Journal of Entrepreneurship in Emerging Economies, 9(1), 85-96 (2017).

Baker, T., y Welter, F., Come on out of the ghetto, please! - building the future of entrepreneurship research, https://doi.org/10.1108/IJEBR-02-2016-0065, International Journal of Entrepreneurial Behavior \& Research, 23(2), 170184 (2017).

Bretones, F. D., y Radrigán, M., Actitudes hacia el emprendimiento: el caso de estudiantes universitarios chilenos y españoles, https://doi.org/10.7203/CIRIEC-E.94.12668, CIRIEC-España, 0(94), 11-30 (2018).

Farhat, S. D., y Guevara, R. Incidencia de la actitud conductual en las intenciones emprendedoras en estudiantes universitarios, https://doi.org/10.22201/fca.24488410e.2019.2074, Contaduría y Administración, 65(2), 1-26 (2019).

Fayolle, A., y Gailly, B., The impact of entrepreneurship education on entrepreneurial attitudes and intention: hysteresis and persistence, https://doi.org/10.1111/jsbm.12065, Journal of Small Business Management, 53(1), 75-93 (2015).

Fernández-Serrano, J., y Liñán, F., Culture and entrepreneurship: the case of latin america, https://doi.org/10.15446/innovar.v24n1spe.47616, Innovar, 24(SPE), 169-180 (2014). 
Fietze, S., y Boyd, B., Entrepreneurial intention of danish students: a correspondence analysis, https://doi.org/10.1108/IJEBR-08-2016-0241, International Journal of Entrepreneurial Behavior \& Research, 23(4), 656672 (2017).

Fragoso, R., Rocha-Junior, W., y Xavier, A., Determinant factors of entrepreneurial intention among university students in Brazil and Portugal, https://doi.org/10.1080/08276331.2018.1551459, Journal of Small Business \& Entrepreneurship, 32(1), 33-57 (2020).

García, J. C. S., Carrizo, A. L., y Yurrebaso, A., Variables determinantes de la intención emprendedora en el contexto universitario, Revista de psicología social aplicada, ISSN: 1131-6225, 15(1), 37-60 (2005).

García-Peñalvo, F. J., La tercera misión, https://doi.org/10.14201/eks2016171718, Education in the Knowledge Society (EKS), 17(1), 7-18 (2016).

Gujrati, D. R., Tyagi, D. V., y Lawan, L. A., Family financial status and students' entrepreneurial intention: the mediatory role of entrepreneurship education, https://doi.org/10.34218/JOM.6.3.2019.003, Journal of Management, 6(3), 21-28 (2019).

Hendrajaya, H., Widodo, J., Rachman, M., y Raharjo, T. J., Determinant model of student entrepreneurship awareness, https://doi.org/10.15294/jed.v7i1.29324, The Journal of Educational Development, 7(1), 1-15 (2019).

Herman, E., Entrepreneurial intention among engineering students and its main determinants, https://doi.org/10.1016/j.promfg.2019.02.220, Procedia Manufacturing, 32, 318-324 (2019).

Jena, R. K., Measuring the impact of business management student's attitude towards entrepreneurship education on entrepreneurial intention: a case study, https://doi.org/10.1016/j.chb.2020.106275, Computers in Human Behavior, 107, 106275 (2020).

Krauss, C., Actitudes emprendedoras de los estudiantes universitarios: el caso de la Universidad Católica del Uruguay, Dimensión empresarial, ISSN: 1692-8563, 9(1), 28-40 (2011).

Krueger, N. F., y Brazeal, D. V., Entrepreneurial potential and potential entrepreneurs, https://doi.org/10.1177/104225879401800307, Entrepreneurship Theory and Practice, 18(3), $91-104$ (1994).

McCann, B. T., Prior exposure to entrepreneurship and entrepreneurial beliefs, https://doi.org/10.1108/lJEBR-05-20160160, International Journal of Entrepreneurial Behavior \& Research, 23(3), 591-612 (2017).

Piirainen, K. A., Andersen, A. D., y Andersen, P. D., Foresight and the third mission of universities: the case for innovation system foresight, https://doi.org/10.1108/FS-04-2014-0026, Foresight, 18(1), 24-40 (2016).

Pinheiro, R., Langa, P. V., y Pausits, A., The institutionalization of universities' third mission: Introduction to the special issue, https://doi.org/10.1080/21568235.2015.1044551, European Journal of Higher Education, 5(3), $227-232$ (2015).

Robinson, P. B., Stimpson, D. V., Huefner, J. C., y Hunt, H. K., An attitude approach to the prediction of entrepreneurship, https://doi.org/10.1177/104225879101500405, Entrepreneurship Theory and Practice, 15(4), 13-32 (1991).

Secundo, G., Elena Perez, S., Martinaitis, Ž., y Leitner, K. H., An intellectual capital framework to measure universities' third mission activities, https://doi.org/10.1016/j.techfore.2016.12.013, Technological Forecasting and Social Change, 123(C), 229-239 (2017).

Soria-Barreto, K., Zuniga-Jara, S., y Ruiz-Campo, S., Educación e intención emprendedora en estudiantes universitarios: un caso de estudio, https://doi.org/10.4067/S0718-50062016000100004, Formación universitaria, 9(1), 25-34 (2016).

Valliere, D., Multidimensional entrepreneurial intent: an internationally validated measurement approach, https://doi.org/10.1108/IJEBR-08-2015-0182, International Journal of Entrepreneurial Behavior \& Research, 23(1), 59-77 (2017).

van Hemmen, S., Urbano, D., y Alvarez, C., Charismatic leadership and entrepreneurial activity: an empirical analysis, Innovar, ISSN 0121-5051, 23(50), 53-65 (2013).

Wardana, L. W., Narmaditya, B. S., y otros cinco autores, The impact of entrepreneurship education and students' entrepreneurial mindset: the mediating role of attitude and self-efficacy, https://doi.org/10.1016/j.heliyon.2020.e04922, Heliyon, 6(9), e04922 (2020).

Zomer, A., y Benneworth, P., The rise of the university's third mission, https://doi.org/10.1007/978-94-6091-555-0_6, en J. Enders, H. F. de Boer, y D. F. Westerheijden (Eds.), Reform of Higher Education in Europe, 81-101, SensePublishers, (2011). 